\title{
La identidad personal en relación con la educación: características y formación del concepto
}

\author{
Alice Bajardi. Universidad de Granada \\ 口 \\ Recepción: 12 de abril de 2015 | Revisión: 30 de mayo de 2015 | Aceptación/Publicación: 24 de julio de 2015 \\ Correspondencia: alicebajardi@ugr.es | http://hdl.handle.net/10481/37124
}

\begin{abstract}
Resumen: El presente artículo, que introduce el número monográfico sobre Identidad y Educación, trata el tema de la identidad personal y proporciona un excursus bibliográfico ilustrando varios puntos de vista tanto filosóficos como psicológicos y pedagógicos. Además, se analizan las distintas fases de la formación de la identidad personal argumentando su relación fundamental con el cuerpo, el espacio, la imagen, la familia, la escuela y con la sociedad. Finalmente se proporciona una opinión personal sobre las características de la identidad personal poniendo de manifiesto la necesidad de proponer y desarrollar el tema de la identidad a partir de la planificación de los proyectos escolares y de los programas de formación docente.
\end{abstract}

Palabras clave: Identidad Personal | Características y Desarrollo Individual | Autoconcepto | Educación

THE PERSONAL IDENTITY IN RELATION TO EDUCATION: CHARACTERISTICS AND FORMATION OF THE CONCEPT

\begin{abstract}
This article, which introduces the special issue on Identity and Education, deals with the topic of the personal identity and provides a bibliographic excursus describing various points of view as the philosophical, psychological and pedagogical ones. In addition, we analyze the different phases of the personal identity formation arguing its fundamental relationship with the body, the space, the image, the family, the school and with the society. Finally, a personal opinion is provided on the characteristics of personal identity pointing out the need to propose and develop the identity theme in school projects and teacher training programs.
\end{abstract}

Keywords: Personal Identity | Individual Development and Characteristics | Self Concept | Education L'IDENTITÁ PERSONALE IN RELAZIONE CON L'EDUCAZIONE: CARATTERISTICHE E FORMAZIONE DEL
CONCETTO

Riassunto: Questo articolo, che introduce il numero monografico "Identità ed Educazione" tratta il tema della identitá personale e fornisce un excursus bibliografico sullidentità, illustrando diversi punti di vista (filosofici, psicologici e pedagogici) sull'argomento. Inoltre, si analizzano le varie fasi di formazione dell' identità personale argomentando la sua fondamentale relazione con il corpo, lo spazio, l'immagine, la famiglia, la scuola e la società. Infine, si espone una opinione personale circa le caratteristiche dell' identità personale, sottolineando la necessità di proporre e sviluppare il tema dell'identità a partire dalla pianificazione dei progetti scolastici e dei programmi di formazione degli insegnanti.

Financiación y agradecimientos: Este trabajo ha sido realizado gracias al apoyo del Dpto. de Didáctica de la Expresión Musical, Plástica y Corporal y al soporte de la Beca del Plan Propio de la Universidad de Granada. Un agradecimiento especial a la Dirección Editorial de ReiDoCrea por invitarme a coordinar el monográfico de Identidad y Educación.

\section{Introducción}

Hay algo de lo que todos los seres humanos tienen una necesidad indiscutible desde que aparecieron en este planeta, la búsqueda de una respuesta a la pregunta: "¿Quién soy yo?".

Según Baumeister (1999), de hecho, para las personas ningún tema es más importante e interesante de las personas mismas y, en particular, el Sí mismo. Activar este proceso de conocimiento es esencial para dar sentido y significado a las propias acciones y a las propias vidas.

Tratar el tema de la identidad comporta tomar conciencia de su multidimensionalidad y complejidad. Pero la heterogeneidad y la relatividad de los numerosos trabajos realizados sobre este tema no nos eximen del estudio y de la observación, e indican 
que la identidad del individuo no puede ser definida de manera completa, definitiva e inmutable. Por eso los autores distinguen diferentes tipos de identidad, como la cultural (Berry 2001), social (Tajfel 2010), nacional (Edensor 2002), religiosa (Mardones, Beriain et al. 1996), sexual (Córdoba García 2003), de género (Litosseliti and Sunderland 2002).

Gere y Crawford (1998) ya habían afirmado que podemos ser caracterizados por diferentes identidades, dependiendo del contexto social, y que existen relaciones entre estas identidades.

Es mejor entonces reconocer que en la definición de identidad existe una pluralidad de sub-identidades. Para expresar este concepto, Mishler (1999) utilizó la metáfora de "nosotros mismos como un coro de voces, no sólo como un tenor o soprano solista."

Los enfoques enumerados anteriormente para definir la identidad se refieren a aspectos objetivos tales como: la identidad social, la identidad física y la identidad psicológica (el estilo de comportamiento y la personalidad de un individuo). "Al igual que todo el mundo tiene una identidad para los demás, también tiene una identidad para sí mismo. La identidad para los demás es la identidad objetiva, la identidad para sí mismo es la identidad subjetiva. La identidad subjetiva es el conjunto de mis características por como las veo y las describo en mí mismo" (Jervis 1998).

El objeto de estudio de este artículo de abertura del número monográfico "Identidad y Educación" es la identidad personal, tomando en consideración la identidad subjetiva y tratando también aspectos de la identidad objetiva porque el modo de vernos es en parte el reflejo de la manera en que nos ven los demás, o que sabemos/ creemos que nos ven los demás. Este trabajo constituye una parte de mi investigación y tesis doctoral en educación artística (dirigida por D. Álvarez Rodríguez y R. Marín Viadel del Departamento de Didáctica de la Expresión Musical, Plástica y Corporal de la Universidad de Granada) que proporciona una recopilación y revisión bibliográfica útil a introducir este número monográfico.

Como investigadora y docente me parece que este tema es de interés fundamental para la educación que ciertamente no puede evitar de abordarlo, de lo contrario se correría el "riesgo de promover y evaluar la apropiación de aprendizaje en el que los individuos no comparten un sentido, no viven como capaces de añadir algo a su identidad personal y valorizarla" (Dallari 2000). Además es totalmente "incongruente la transmisión del conocimiento separado de la posibilidad de verlo y utilizarlo de manera crítica", por lo que necesitamos hacer nuestros los conocimientos y las habilidades adquiridas a través de nuestro propio estilo de aprendizaje, enriqueciendo así nuestra propia identidad cultural (Dallari 2000).

\section{EI SÍ MISMO Y LA IDENTIDAD}

Al igual que en literatura no encontramos un sentido único y determinado del concepto de identidad, de la misma manera las definiciones atribuidas al concepto del sí mismo son numerosas y no son unívocas. Acercarse al estudio de la identidad y del sí mismo es, sin duda, un camino complejo y orientarse en la búsqueda de un denominador común entre los autores es arduo. En todo caso este tema ha sido tratado ampliamente desde la psicología y el psicoanálisis pero aún antes desde la filosofía.

Según algunos autores del campo de la psicología los términos identidad y sí mismo deben permanecer conceptos distintos (Alsaker and Kroger 2006). Por ejemplo, 
Baumeister (1986) diferencia estos dos términos especificando que el sí mismo es la descripción de quien uno es, mientras la identidad es la definición de quién uno es. Otros autores, en cambio, afirman que los dos conceptos están estrechamente interconectados (Leary and Tangney 2003) y no siempre son distinguibles (Castiglioni 1994).

Algunos autores prefieren utilizar el término sí mismo, cuando se hace referencia a la persona como un ser consciente y con capacidad de auto-reflexión, mientras que otros emplean el término identidad para indicar a la persona en su totalidad y unicidad. Según Olson (2003) no podría ser aceptado el empleo del término sí mismo como sinónimo de persona en su totalidad, en cuanto no deberíamos decir "que la persona es el sí mismo sino más bien que la persona tiene un sí mismo". McCormick y Pressley (1997) afirman que nuestro concepto de sí mismo puede ser definido como una representación organizada de nuestras teorías, actitudes y creencias sobre nosotros mismos. En literatura también encontramos los términos yo y mí, que a menudo se refieren respectivamente al sí que conoce como sujeto y el sí conocido como objeto.

Es interesante notar que en el campo de la filosofía ya los presocráticos se habían enfrentado al problema de la unicidad del ser en devenir y, el propio Sócrates, el del conocimiento del sí mismo. En particular Heráclito, entre el VI y el V siglo a. C., partiendo de la idea homérica de psychè buscó el principio que uniera la vida del cuerpo con la vida mental comprensiva de sensaciones y emociones. Pero los filósofos posteriores no desarrollaron esta intuición y la dirección fue considerar la psique de manera incorpórea, luego identificándola definitivamente con el alma, que quedó la categoría de referencia para entender nuestro ser y el sentido de la vida de cada individuo.

Sólo a partir del Renacimiento podemos encontrar algunas corrientes de pensamiento que comenzaron a desacreditar la creencia de un núcleo sustancial y permanente de la identidad subjetiva identificable con el alma, por ejemplo la teoría cartesiana de res cogitans y de res extensa y el espiritualismo de Berkeley, aunque se mantuvo constante el pensamiento de considerar el centro del ser del sujeto una cosa espiritual, un yo dado e idéntico a sí mismo incluso en situaciones cambiantes.

Kant en sus postulados de la Crítica de la Razón Práctica (1788) escribe sobre el yosustancial que debe ser reemplazado por las actividades del yo-pienso, como función lógica de auto-conciencia, gracias a la cual conocemos y sabemos de conocer. Kant a diferencia de Descartes, postula que no es la razón la que lo lleva a sus conclusiones sino que es la fe y la necesidad de la existencia de la moral humana absoluta y universal para que el hombre tenga un alma inmortal. Kant argumenta que el límite de nuestra razón podemos explicarlo cuando, por ejemplo, contemplamos una obra de arte y nos acercamos a ella "sintiéndola"; en este caso, superaríamos los límites de nuestra razón y de lo que podemos saber.

Posteriormente, los románticos usaron expresiones como "alma del mundo o "espíritu del mundo" que se puede encontrar tanto en la naturaleza como en nuestra alma, como un "yo" que crea las cosas del mundo. Hegel desarrolló la dialéctica del "en sí" y del "por si" con el poder autoreflexivo del espíritu. Antes el espíritu se pone consciente de sí en el individuo (espíritu subjetivo) y luego alcanza una mayor conciencia en la familia, en la sociedad y en el Estado (espíritu objetivo). Es con el espíritu absoluto (que incluye en sí mismo toda la evolución de los seres humanos) que el espíritu alcanza la más alta forma de auto-conciencia, como en el arte, en la religión y, sobre todo, para Hegel, en la filosofía. Si, según Hegel, el ser humano llega a la conciencia de sí mismo a través del conflicto y la contradicción, según Feuerbach, lo consigue a 
través de las relaciones de amistad, de amor y de todas las formas de interacción basadas en el diálogo.

Después de esta sumaria exposición sobre la búsqueda del sí basada sobre una parte de la filosofía dónde principalmente el sí correspondía al alma entendida como núcleo central e indivisible, es oportuno volver a recordar que también la psicología ha contribuido a aumentar nuestro conocimiento ejerciendo con la introspectiva búsqueda del sí una notable influencia sobre la cultura del siglo XX en adelante. La psicología, en todo caso, nos proporciona una definición poco exhaustiva de los conceptos de sí mismo y de identidad, confundiendo y/o sobreponiendo a menudo los dos términos. Podemos sintetizar los diferentes puntos de vista sobre el sentido del sí en dos grandes escuelas de pensamiento: quien cree que el sí esté presente ya desde el nacimiento y quién, en cambio, afirma que se trate de una construcción social (Bombi and Pinto 2000).

\section{LA IDENTIDAD PERSONAL}

John Locke (1632-1704), en su "Ensayo sobre el entendimiento humano" (1690), fue el primero en hablar de la identidad personal, justo cuando entró en crisis la idea metafísica y religiosa del alma, entendida como sustrato unitario que garantiza la continuidad de las experiencias. Acabada la idea de un yo inmutable (del alma inmortal) la continuidad del yo se reduce a un "hilo de la memoria". David Hume (1711 -1776) evidencia aún más este elemento de fragilidad considerando la identidad una cosa construida, ficticia o, más bien, un "fetiche" (Bodei, Cantillo et al. 2004), no más que una colección de diferentes y veloces percepciones en continuo movimiento.

En la actualidad el concepto de identidad tiene en literatura diferentes significados y definiciones, aunque éstos tienen en común la idea de que la identidad no es un atributo fijo de una persona, sino un fenómeno relacional. La psicóloga Erikson (1968), de hecho, explica que la identidad no es algo que se tiene, sino algo que se desarrolla durante toda la vida. En este contexto, entonces, la identidad puede figurarse como una respuesta a la pregunta recurrente: "¿quién soy yo en este momento?" (Beijaard, Meijer et al. 2004).

El pedagogo Marco Dallari (2000), escribe que "la identidad no es el sujeto y tampoco está en el sujeto. La identidad está delante, detrás y alrededor de nosotros, está en la relación y en el intercambio". No podemos ver la identidad, sino observar "los modos de manifestarse de la identidad y, a través de esta, tratar de entender/construir algo de nosotros mismos" (Dallari 2000).

Según Giovanni Jervis (1998), la identidad es reconocerse y ser reconocidos, es lo que caracteriza a cada uno como individuo único e inconfundible. También Núñez Ladezéve (2005), define la identidad personal como el conjunto de condiciones que le permiten a una persona de considerarse un ser humano par y diferente de los otros, un ser de una especie diferente de todas las otras. Según Alvarez Munárriz (2011), en cambio, podríamos describirla como la conciencia y la asunción de modos de ser, de pensar y de actuar que da sentido y objetivo a la vida de un individuo.

Por último, creo que habría que reconocer en la definición de identidad la existencia de una pluralidad de sub-identidades. Explica muy bien este concepto Mishler (1999), utilizando la metáfora de "nosotros mismos como un coro de voces, no sólo como tenor o soprano solista". También Gee y Crawford (1998), han afirmado que podemos ser caracterizados por identidades diferentes según el contexto social y que existen relaciones entre estas identidades. 


\section{LA IDENTIDAD PERSONAL EN RELACIÓN CON EL CUERPO Y EL ENTORNO}

A lo largo de la vida conocemos el mundo a través del cuerpo, pero esta dimensión natural de la identidad que está vinculada a la adquisición de la conciencia del cuerpo, en realidad, es profundamente arraigada en la cultura de pertenencia, los hábitos, los modelos e las ideologías compartidas. La relación entre el cuerpo y la identidad es "extraña, frágil y compleja" (Dallari 2000) y cambia en el tiempo. El niño toma lentamente conciencia del propio cuerpo y del espacio, de su imagen refleja en el espejo o refleja en la mirada de los demás. Luego, llega el momento en que pregunta al cuerpo de ser imagen identitaria del contexto cultural e histórico en que vive y al que quiere identificarse. De aquí la importancia de la familia, de la escuela y de los encuentros en la sociedad. "La identidad crece y se consolida a través de contratos e instituciones que nos ofrecen la oportunidad de llegar a un acuerdo con los demás y de negociar el reconocimiento del sí", (Dallari 2000). Así, incluso antes de tomar conciencia de la propia sexualidad, el niño aprende su nombre, con el que se reconoce y se hace reconocer (Quién eres? ¿Cómo te llamas?) y, luego, el apellido, con el que coge conciencia de pertenecer a un grupo familiar pero no sólo desde el punto de vista afectivo, sino también como vínculo institucional y simbólico. También la envoltura externa como los vestidos y los accesorios que llevamos hablan de nuestra identidad y sirven para hacernos reconocer (Bajardi 2014).

La identidad no es un objeto que es posible aislar y definir con seguridad, y el ambiente es un elemento que indudablemente la influencia como el contexto histórico y socio-cultural en el que la gente vive y afirma su identidad. Álvarez-Munárriz (2011), explica la diferencia entre identidad social e identidad personal, donde la primera se refiere a una sensación de similitud con los demás, mientras que la segunda se refiere a un sentimiento de diferencia con respecto a los demás. Se destaca, sin embargo, que ambos tipos de identidad forman parte de un conjunto unitario, que son partes inseparables y que se combinan y se mezclan, en el sistema dinámico y relacional que es la persona. Hablando de la persona como sujeto contextual (no puede existir sino en relación con los demás), Bateson (1979) explica este concepto utilizando la metáfora del leñador que acepta un árbol. El autor señala que la acción de un solo componente del sistema (el leñador que golpea el tronco con el hacha) modifica todos los componentes implicados. Así, el hombre cambia su posición en el espacio reforzando su musculatura y desgastando sus articulaciones, el hacha consume su filo y el árbol cae al suelo partiéndose. El concepto de sujeto contextual, en fin, nos recuerda como una relación sea capaz de modificar y también influenciar a todos sus participantes, incluso cuando la acción empieza a partir de sólo uno de ellos; ésta es la base para afirmar que el individuo construye su propia identidad en la relación e interacción con los demás y con su entorno.

\section{EL PROCESO DE FORMACIÓN DE LA IDENTIDAD}

El desarrollo de la identidad se produce en un campo intersubjetivo y puede ser definido como un proceso continuo, un proceso de interpretación de sí mismos como un cierto tipo de persona y de reconocimiento como tal en un determinado contexto (Gee 2001).

Pérez de Lara Ferré (2001) señala que las personas son seres definidos y en constante construcción y cambio y que debemos tener en cuenta varios factores de la dimensión pública o social en la construcción de la identidad, en cuanto elemento llave de la realidad subjetiva y, como tal, en relación dialéctica con la sociedad. 
La formación de la identidad está relacionada con diferentes procesos de desarrollo basados en la evolución progresiva de la imagen de sí o de la representación de sí durante toda la vida de un individuo, lo que le permite sentir un "sentido de identidad consigo mismo" (Aleni Sestito 2004) y teniendo en cuenta los muchos cambios relacionados con sí mismos y con los demás. Estos diferentes procesos de la evolución de la identidad están caracterizados por cuatro componentes, o sea la identificación, la individuación, la imitación y la internalización.

La identificación es un proceso por el cual el individuo forma su personalidad asimilando y modelado sobre sí algunos rasgos de otra persona al que se siente parecido y con la cual comparte algunos caracteres. Eso produce un sentido de pertenencia a una entidad colectiva definida como "nosotros", por ejemplo, en sentido creciente de lo micro a lo macro, la familia, un grupo de pares, la comunidad local, la nación hasta la entera humanidad. Esta fase de identificación se relaciona con el proceso de inclusión.

El proceso de individuación nos permite localizar a las características que nos distinguen de otras personas ya sean internas o externas al grupo al que se siente pertenencia. La persona siente que puede detectar la diferencia entre sí y los demás a través de sus propias características y su historia personal. Se relaciona esta fase de individuación para el proceso de exclusión.

A través de la imitación, comportamiento, antes instintivo y luego reflejo, de reproducción de modelos conductuales, el individuo se mueve de manera distinta en la sociedad según el contexto social en que se encuentra. El concepto de imitación constituye el núcleo de la psicología social del criminólogo, sociólogo y filósofo Gabriel Tardos (1843-1904) que consideró este fenómeno fundamental en la constitución de las relaciones sociales.

Finalmente, el proceso de la interiorización permite al individuo crear una imagen de sí mismo gracias a la importancia que tienen las actitudes, las opiniones, los valores y los comportamientos de los demás sobre sí.

Según Erikson (1968) las fases de la adolescencia y la juventud de una persona representan períodos naturales fundamentales en los que es posible "afrontar y solucionar positivamente uno de los conflictos vitales que caracteriza el desarrollo a lo largo del curso de la vida, del nacimiento al vejez" (Aleni Sestito 2004). Además Erikson, que define la identidad del yo como un estado interior del individuo resultante de la función de síntesis del yo, cree que esta se desarrolla a partir de la conciencia de coherencia y continuidad del propio sentido por sí mismo y por los demás. De hecho, Erikson, creyendo que el desarrollo del yo está relacionado con las variables socioculturales, analiza las posibles interacciones entre el microcosmos individual y el macrocosmos social.

Cada momento del desarrollo se compara con las características, modelos y objetivos culturales del grupo social de pertenencia así que el proceso de maduración individual termina coincidiendo con el proceso de socialización. Por tanto, el modelo eriksoniano de la maduración prevé que la evolución individual sólo pueda conseguir resultados armónicos si los continuos cambios determinados por el impulso innato al desarrollo encuentren en el grupo de referencia esquemas de comportamiento actos a inscribir en la cultura común las urgencias individuales, que las diversas etapas y las crisis del desarrollo implican. 


\section{Conclusiones}

Este trabajo pone de manifiesto como la identidad sea un tema fundamental en diferentes campos, como la filosofía, el arte, la psicología, la sociología y finalmente la educación que es nuestro campo de investigación. Mientras que antiguamente se relacionaba la identidad con el alma entendida como núcleo central, un elemento estático e indivisible, actualmente la identidad se concibe como muy complejidad, dinámica y sobre todo multidimensional. Además se ha introducido la concepción de la identidad como un fenómeno relacional y no como un atributo fijo de una persona. De hecho, se cree que el individuo construya su propia identidad a partir de su integración y relaciones con los demás y el ambiente.

El filósofo francés Gilles Deleuze, a partir de la teoría de Hume de que la identidad era una mera ficción, apoyó la idea de variación continua de la identidad, es decir, que nadie es el mismo sí mismo del instante anterior o del instante después. Sin embargo esta visión muy dinámica de la identidad en mi opinión supone un cambio considerable de los puntos de vista y de las normas de nuestra sociedad actual; en caso contrario llevaría a grandes incongruencias. De hecho, siguiendo el punto de vista deleuzeiano un asesino que ha cometido un acto criminal hoy, mañana no podría ser juzgado en un tribunal por aquel acto porque ya no sería el mismo individuo que lo cometió. En efecto, aunque coincido en pensar que la identidad está en constante cambio, creo que existe una pluralidad de sub-identidades y una relación importante entre estas.

Esto me recuerda el trabajo de un alumno con discapacidad muy grave que enfrentándose a un ejercicio de educación artística sobre la identidad realizó un trabajo compuesto por una poesía y una imagen que describían el estado (alegre y esperanzado) de transformación de un oruga en mariposa. La etapa del ser oruga y la del ser mariposa representarían dos identidades distintas, pero probablemente la una no podría existir sin la otra, o una contiene la otra y viceversa.

El tema de la identidad es tratado tanto en la educación formal como en la educación no formal e informal. Sin embargo, considero importante hacer hincapié en la necesidad no sólo de abordar este tema durante breves y ocasionales momentos educativos, sino también de planificar y desarrollar el tema de la identidad a partir del diseño de los proyectos y de los programas educativos para promover la apropiación de aprendizajes significativos, con sentido para los estudiantes. Esto hace que ellos vivan los aprendizajes con capacidad de desarrollar y valorizar su identidad personal.

Esto se aplica no sólo a los estudiantes sino también a los docentes. De hecho lo que el docente cree acerca de la enseñanza y del aprendizaje, y sobre todo de su imagen como educador es de gran importancia; es la base para la producción de sentido / significado y para el proceso de toma de decisiones. Por lo tanto, como profesores e investigadores universitarios, así como educadores de cualquier nivel y contexto educativo, debemos tener en cuenta que la formación docente debería iniciar explorando el sí como educador, con el fin de facilitar los varios procesos de evolución de la identidad docente y prepararnos a la importante tarea de guiar a los estudiantes en su proceso de formación de la identidad personal.

\section{Referencias}

Aleni Sestito, L. (2004). Lo sviluppo dell'identità nella prospettiva del ciclo di vita. Processi di formazione dell'identità in adolescenza. Napoli, Liguori: 33-59. 
Alsaker, F. D. and J. Kroger (2006). Self-concept, self-esteem and identity. Handbook of adolescent development. S. Jackson and L. Goosens. New York, Psychology Press: 90-117.

Álvarez-Munárriz, L. (2011). "La compleja identidad personal." Revista de dialectología y tradiciones populares 66(2): 407-432.

Bajardi, A. (2014). Construyendo Identidades en la Investigación Educativa Basada en las Artes. (Re)Presentaciones, Miradas, Reflejos en Investigación Basada en Artes y en Investigación Artística. R. Marín Viadel, J. Roldán and J. Mena de Torres. Granada, Universidad de Granada. 3: 93-103.

Bateson, G. (1979). Mind and Nature: a necessary unity. New York, Dutton.

Baumeister, R. F. (1986). Identity: Cultural change and the struggle for self, Oxford University Press.

Baumeister, R. F. (1999). The self in social psychology. Philadelphia, Psychology Press.

Beijaard, D., P. Meijer, et al. (2004). "Reconsidering research on teachers." Teacher and Teacher Education 20: 107128.

Berry, J. W. (2001). "A psychology of immigration." Journal of social issues 57(3): 615-631.

Bodei, R., G. Cantillo, et al. (2004). Ricostruzione della soggettività. Napoli, Liguori.

Bombi, A. S. and G. Pinto (2000). Le relazioni interpersonali del bambino. Studiare la socialità infantile con il disegno. Roma, Carocci.

Castiglioni, M. (1994). II Sé e l'identità. Psicologia sociale e dello sviluppo - Parte I: Psicologia Sociale. C. Castelli, A. Quadrio and L. Venini. Milano Franco Angeli: 173-217.

Córdoba García, D. (2003). "Identidad sexual y performatividad." Athenea digital: revista de pensamiento e investigación social 4: 087-096.

Dallari, M. (2000). I saperi e l'identità: costruzione delle conoscenze e della conoscenza di sé. Milano, Guerini e Associati.

Edensor, T. (2002). National identity, popular culture and everyday life. Oxford - New York, Berg.

Erikson, E. H. (1968). Identity: Youth and crisis. New York, Norton \& Company.

Gee, J. P. (2001). Identity as an analytic lens for research in education. Review of research in education. W. G. Secada. Washington, DC: American Educational Research Association. 25: 99-125.

Gee, J. P. and V. M. Crawford (1998). Two kinds of teenagers: Language, identity and social class. Reconceptualizing the literacies in adolescents' lives. D. Alvermann, K. Hinchman, D. Moore, S. Phelps and D. Waff. Mahwah, NJ, Lawrence Erlbaum Associates Publishers: 225-245.

Jervis, G. (1998). "Che cos' è l'identità." Enciclopedia Multimediale delle scienze filosofiche.

Kant, I. (1788). Kritik der praktischen Vernunft.

Leary, M. R. and J. P. Tangney (2003). Handbook of self and identity, Guilford Press.

Litosseliti, L. and J. Sunderland (2002). Gender identity and discourse analysis. Amsterdam, John Benjamins Publishing.

Locke, J. (1690). Saggio sull'intelletto umano.

Mardones, J., J. Beriain, et al. (1996). La identidad religiosa en la modernidad actual. Identidades culturales. J. y. L. Beriain, P. Bilbao, Deusto.

McCormick, C. B. and M. Pressley (1997). Educational psychology: Learning, instruction, assessment. New York, Longman Publishing/Addison Wesley L. 
Mishler, E. G. (1999). Storylines: Craft artists' narratives of identity. Cambridge, Harvard University Press.

Núñez Ladezéve, L. (2005). Identidades humanas: conflictos morales en la postmodernidad. Madrid, Centro de Estudios Políticos y Constitucionales

Olson, E. T. (2003). Personal Identity. The Blackwell dictionary of political science: a user's guide to its terms. F. Bealey, Wiley-Blackwell: 352-368.

Pérez de Lara Ferré, N. (2001). Identidad, diferencia y diversidad: mantener viva la pregunta. Habitantes de babel. Política y Pedagogía de las diferencias. C. y. L. Skliar, J. Barcelona, Laertes.

Tajfel, H. (2010). Social identity and intergroup relations, Cambridge University Press. 